\title{
The Research In MAC Protocol Of WBAN Based On The Data Priority Of Medical Applications
}

\author{
JieWANG ${ }^{1}$, BiZENG ${ }^{2}$ \\ ${ }^{1}$ School of Computer Science, Guangdong University of Technology, China \\ ${ }^{2}$ School of Computer Science, Guangdong University of Technology, China
}

Keywords: WBAN, MAC Protocol, priority

\begin{abstract}
With the development of wireless sensor networks, the application of the human body area network wireless draws people's attention, especially in the medical field. Due to the limited node energy which is not easy to replace, it is so important to reduce node energy consumption and prolong the lifetime of the network nodes. In medical applications, data type and amount of data size is significantly different. It is a good strategy that we Set priorities to improve the success rate, reduce collisions, in order to save energy.
\end{abstract}

\section{Introduction}

Wireless body area network (WBAN) refers to monitor various parts of the body physiological parameters (such as temperature, ECG, blood pressure, EMG, heart rate, oxygen concentration, etc.) by the human body or the surface of various special sensors, and then the center node will transmit the data to a remote monitoring center by a wireless network. It is not only the new health care, disease surveillance solutions, but also the perception of things and part of the group. May 2010, IEEE 802.15.6 working group creates a first draft, the document gives the physical layer, MAC layer standards, the development prospects of wireless body area networks attract more researchers participation.

Some wireless body area network nodes implanted battery cannot be frequently replacement, the node energy consumption has become a key research point in wireless body area networks. Studies have shown that the main factor for node energy consumption is channel listening when sending frame, retransmission after the frames collide. The MAC layer protocol is to control a channel access, reduce the collision of node data frame, and reduce the retransmission rate and network delay, to ensure the reliability of communications and nodes of the energy efficiency.

\section{WBAN Medical Applications}

The different sensors which are in the body or surface to detect human physiological conditions, such as: blood pressure, blood oxygen, electrocardiogram (ECG), body temperature, breathing machine, electroencephalogram (EEG), electromyography (EMG) and so on. The development of medical technology, new implantable medical sensors have been applied.

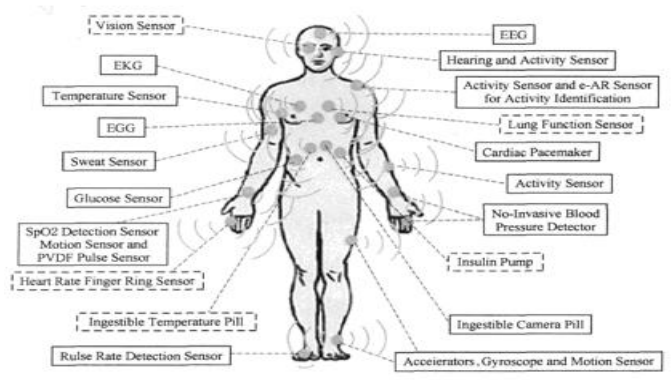

Fig1.WBAN for medical applications

In a body area network, due to the use of multiple sensors, and the difference between the amount of data sent and priority cases, resulting in significant business diversification. 


\begin{tabular}{l|c|c|} 
& Sampling Frequency $(\mathrm{HZ})$ & Data Rate(Kbps) \\
\hline Blood pressure & 60 & 1.44 \\
\hline ECG & 250 & 72 \\
\hline EEG & 200 & 920 \\
\hline Temperature & 0.1 & 2.4 \\
\hline Blood Oxygen & 300 & 7.2 \\
\hline video & 25 & $384-700$ \\
\hline
\end{tabular}

Fig2. WABN data reference table

The data of ECG, EEG, video are large number the others, such as blood pressure, body temperature, blood oxygen are small amount of data. ECG, EEG, blood pressure needed is real time data, so they are higher priority.

\section{WBAN MAC Protocol}

In the IEEE802.15.6 standard, the channel is divided into super-frame structure, a sink node periodically broadcasts a beacon frame, and in the time between two beacon frames is called a superframe. Each super-frame consists of the same length as the boundary beacon period. Hub selected periodic boundary beacons in order to select the slot allocation. Hub as the central node is responsible for selecting one of the following three access methods, and to establish a coordinated channel access. Superframe structure IEEE802.15.6 in the following three operating modes:

beacon mode with super frame boundary In this mode, Hub should be active in each beacon period divided applicable access phase, shown in Figure 1-3, displays IEEE802.15.6 super-frame structure , super-frame structure is divided into exclusive access stage 1 (EAP1), random access phase 1 (RAP1), Type I / II stage, exclusive access stage 2 (EAP2), random access phase 2 (RAP2), Type I / phase II, as well as a competitive access phase (CAP).

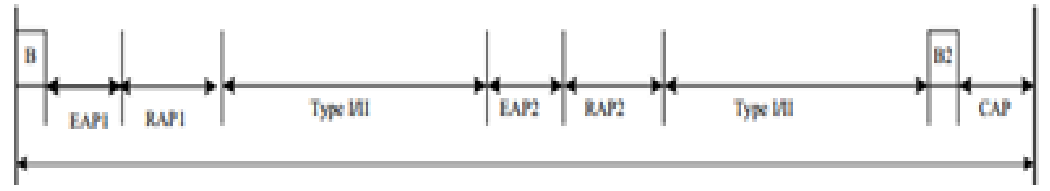

Fig3. IEEE 802.15.6 Superframe structure

EAP, RAP and CAP stage, node uses CSMA / CA or slot Aloha access to competitive resource allocation.EAP1 and EAP2 for highest priority traffic, and RAP1, RAP2 and CAP only used for normal traffic. Type I / II stage for upstream, downstream, two-way and two-way delay time interval distribution. In Type I / II stage, polling is used as the allocation of resources. Depending on the application requirements, the center can continue by setting the length to 0 to disable any of these stages.

2) non-beacon mode and super-frame boundary

In this mode, the entire super-frame time is covered by a Type I or Type II.

3) non-beacon mode with no super-frame boundary

In this mode, the coordinator polling only occasional Type II distribution.

\section{The Proposed WBAN MAC Protocol}

The presence of a diversified business in wireless body area network, the MAC layer protocol optimizes for different business data, important data on the relationship of vital signs such as ECG give high priority class, high-priority data is able to get more slot transmission. Meanwhile, since the wireless body area network data are in the presence of significant relevance, e.g., abnormal physical condition of the patient, blood pressure, body temperature, ECG data may appear abnormal, and different priority can effectively avoid collisions when a node transmits data.

Set the priority criteria are as follows: 
High Priority: including blood pressure, ECG, EEG, such data always changes, and the relatively large amount of data, correlation data between large, real-time requirements. EAP1, EAP2 stage transmission

Low priority: including body temperature, blood oxygen. RAP1, RAP2 and CAP stage transmission.

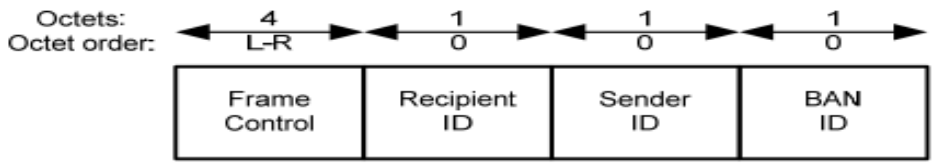

Fig4.MAC Head format

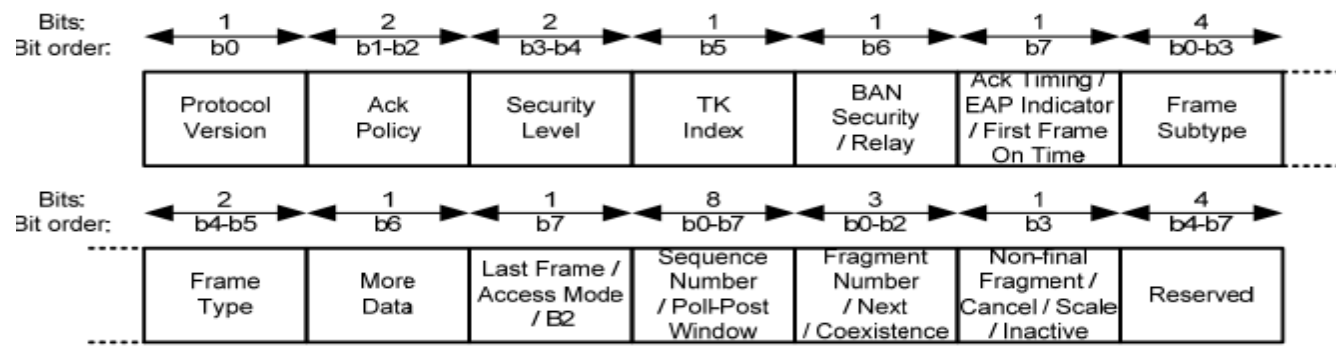

Fig5.Frame Control format

Select the b7 (reserved) in the specific design of as a priority, high priority is 1 and low priority is 0 .

\section{Simulation}

Using OPNET simulation software, the network topology is a star.

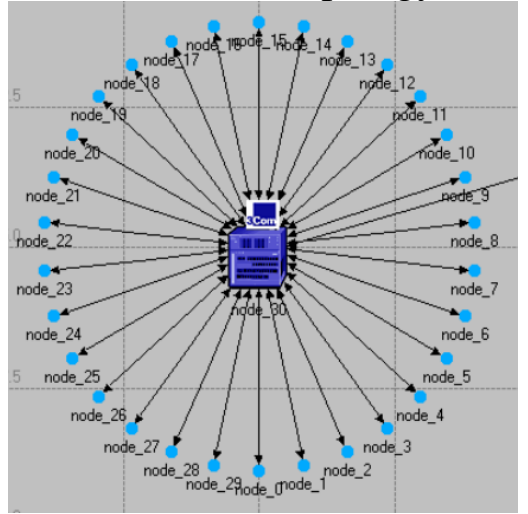

Fig6. The simulation node

The number of nodes is 30 , node $1-15$ is a high priority and low priority node16-19.Node30 is Hub.

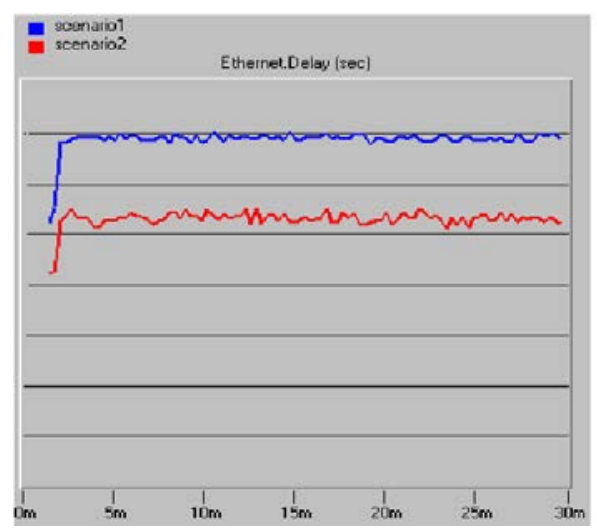

Fig7. Simulation of time delay 
Using a priority MAC, delay of the network reduces and the network performance is improved.Node collision rate decreased, the number of retransmissions reduce,node energy consumption will be reduced.

\section{Summary}

On the basis of the existing research, the paper proposed node priority strategies to reduce collision node,save the node energy . With the development of WBAN, the study of MAC protocol put forward a new direction such as differentiated services, cross-layer design, technology will be more mature, efficient.

\section{References}

[1]IEEE P802.15.6/D06, Wireless Medium Access Control(MAC)andPhysicalLayer(PHY) Specifications for Wireless Personal Area Networks(WPANs) used inor around a body. October 2011.

[2] IEEE Standards Association, IEEE Standard for Local and metropolitan areanetworks-Part 15.6: Wireless Body Area Networks. February 2012.

[3] Kyung Sup Kwak, Sana Ullah and Niamat Ullah, an Overview of IEEE 802.15.6Standard. IEEE 2010.

[4] Injong Rhee, Ajit Warrier and Mahesh Aia, Z-MAC: a Hybrid MAC for WirelessSensor Networks. IEEE/ACM Transactions on Networking, Vol.16, No.3, June2008.

[5] Yuriy Tselishchev, Lavy Libman and Athanassios Boulis, Energy-EfficientRetransmission Strategies under Variable TDMA Scheduling in Body AreaNetworks. 36th Annual IEEE Conference on Local Computer Networks. 2011.

[6] Yuriy Tselishchev, Lavy Libman and Athanassios Boulis, Reducing TransmissionLosses in Body Area Networks using Variable TDMA. IEEE. 2011. 